\title{
Biomechanical Stability of Open-Wedge High Tibial Osteotomy: Comparison of Two Locking Plates
}

\author{
Kota Watanabe ${ }^{*}$, Tomoaki Kamiya², Daisuke Suzuki ${ }^{3}$, Hidenori Otsubo², \\ Atsushi Teramoto², Tomoyuki Suzuki², Toshihiko Yamashita ${ }^{2}$ \\ ${ }^{1}$ Department of Physical Therapy, Sapporo Medical University School of Health Science, Sapporo, Japan \\ ${ }^{2}$ Department of Orthopaedic Surgery, Sapporo Medical University School of Medicine, Sapporo, Japan \\ ${ }^{3}$ Department of Musculoskeletal Biomechanics and Surgical Development, Sapporo Medical University, \\ Sapporo, Japan \\ Email: wkota@sapmed.ac.jp
}

Received 23 July 2014; revised 8 September 2014; accepted 24 September 2014

Copyright (C) 2014 by authors and Scientific Research Publishing Inc.

This work is licensed under the Creative Commons Attribution International License (CC BY). http://creativecommons.org/licenses/by/4.0/

(c) (i) Open Access

\begin{abstract}
Introduction: Recently, new plates with locking screws have been developed and used for medial open-wedge high tibial osteotomy (HTO). The purpose of this study was to evaluate and compare biomechanical properties of different internal fixations in open-wedge HTO using the two currently available locking plates. Methods: Eight paired fresh-frozen cadaveric lower extremities were vertically embedded in steel boxes. The axial compression load was applied to the legs using the mechanical testing machine. The axial compression load test from $0 \mathrm{~N}$ to $550 \mathrm{~N}$ and the failure test were performed before and after HTO. One side of the leg of a specimen was fixed with the Puddu locking plate and the other side was fixed with the TomoFix plate to compare the two plates using the same specimen. A mode of failure and vertical displacement of the medial and lateral parts of the tibia at the osteotomy gap was recorded using a video camera in the failure test. The load-displacement data were analyzed to calculate stiffness, failure load, and displacement at failure. Results: The mean failure load was $1471.4 \mathrm{~N}$ and $1692.3 \mathrm{~N}$ and total vertical displacement at failure was $3.1 \mathrm{~mm}$ and $2.9 \mathrm{~mm}$ with the Puddu and TomoFix plates, respectively. During axial compression loading, displacements mainly occurred at the lateral osteotomy gap, while the medial gap was well preserved. No significant differences were observed in the failure load, displacement, or mode of failure between the two plates. Conclusions: The Puddu and TomoFix plates had similar biomechanical properties in open-wedge HTO. The results indicated reliable stability after open-wedge HTO without fibular osteotomy.
\end{abstract}

\footnotetext{
"Corresponding author.
} 


\section{Keywords}

\section{High Tibial Osteotomy, Puddu Plate, TomoFix, Cadaver, Biomechanics}

\section{Introduction}

High tibial osteotomy (HTO) is an effective and established method in treating osteoarthritis of the medial compartment of the knee. This procedure corrects varus malalignment of the knee, and several techniques of osteotomy have been reported, including close-wedge, open-wedge, and dome osteotomies.

Recently, new plates with locking screws have been developed and used for medial open-wedge HTO. The recent increase in the number of publications on medial open-wedge HTO suggests a renewed interest in this procedure. Two widely used plates include the TomoFix, which is a titanium long plate, and the Puddu plate, which is short with a block incorporated; favorable postoperative results were reported [1] [2]. These plates are believed to achieve more rigid fixation compared with conventional plates, and several biomechanical studies have reported on the mechanical properties of each plate. However, the number of basic studies about biomechanical stability or comparison of the plates is not adequate and previous studies have some limitations such as usage of artificial bones or cadaveric shanks without the fibula. The purpose of this study was to evaluate and compare the biomechanical properties of different internal fixations in open-wedge HTO using the two currently available locking plates.

\section{Material and Method}

\subsection{Specimens}

Eight paired fresh-frozen cadaveric lower extremities (16 legs) were studied. The mean age of the specimens was 82.1 years ( 59 - 92 years). Two were females and 6 were males. The institutional research ethics committee reviewed and approved the study.

The specimens were amputated at the proximal end of the tibia and above the ankle joint. Soft tissues were removed, except for the ligaments, and the specimens were vertically embedded in steel boxes with steel wires and polymethylmethacrylate cement. A specially designed metal plate with a metal sphere was fixed on the proximal surface of the tibia with polymethylmethacrylate cement. Vertical loads could be applied at the tibial lateral plateau through the metal device. The specimens were placed in a mechanical testing machine (AG-X, Shimazu, Japan), which could measure the displacement and the applied loads simultaneously with a mounted load cell while applying the vertical load precisely.

\subsection{Surgical Procedure}

The two different locking plates were used for open-wedge HTO. The spacer plate (Puddu locking plate, Arthrex Inc., Naples, FL, USA) (Figure 1(a)) was a short titanium plate with a spacer to open the osteotomy space. The TomoFix plate (Synthes Gmbh, Oberdorf, Switzerland) (Figure 1(b)) was a long T-shaped titanium plate. A standardized medial open-wedge osteotomy technique was used, with the lateral tibial cortex spared. Oblique osteotomy started at the medial part of the tibia $40 \mathrm{~mm}$ distal to the medial tibial plateau. Osteotomy extended to the lateral proximal part of the tibia $20 \mathrm{~mm}$ distal to the lateral plateau, leaving $10 \mathrm{~mm}$ of lateral bone intact. A standardized opening of $10 \mathrm{~mm}$ was created in all specimens. No bone or materials were inserted in the open space.

\subsection{Biomechanical Test}

Using the mechanical testing machine, the axial compression load was applied to each intact bone at the lateraltibial plateau through the metal sphere on the metal plate from $0 \mathrm{~N}$ to $550 \mathrm{~N}$ at a rate of $1 \mathrm{~mm} / \mathrm{min}$. This testing was performed in three trials after pretesting. Subsequently, HTO was performed and the same test was repeated. Finally, the compression load was applied at a rate of $1 \mathrm{~mm} / \mathrm{min}$ until failure. One side of the leg of a specimen was fixed with the Puddu locking plate and the other side was fixed with the TomoFix plate in order to compare 


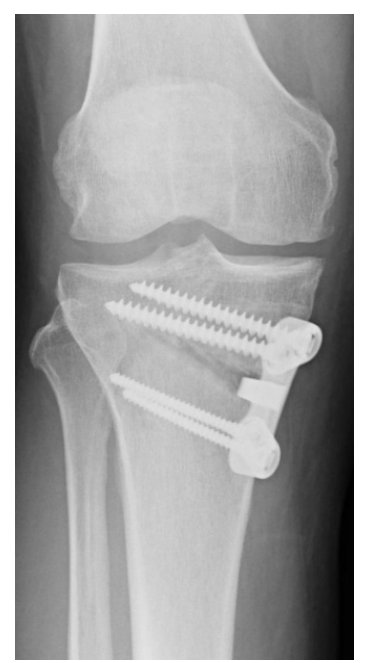

(a)

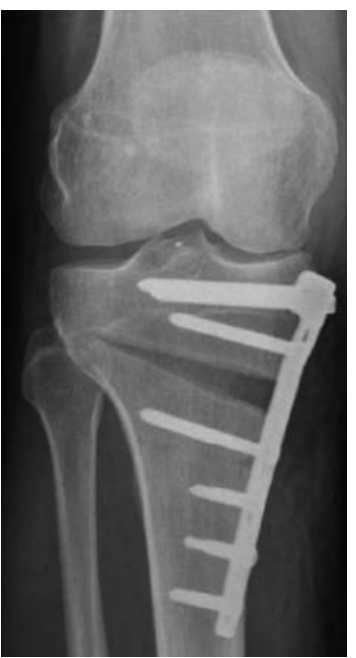

(b)

Figure 1. Radiographs of the clinically used plates for open-wedge HTO: (a) Puddu locking plate (length: 48 - $60 \mathrm{~mm}$, width: $23 \mathrm{~mm}$, diameter of screw: $6.5 \mathrm{~mm}$ (cancellous), $4.5 \mathrm{~mm}$ (cortical)); (b) TomoFix plate (length: $115 \mathrm{~mm}$, proximal width: $33 \mathrm{~mm}$, shaft width: $16 \mathrm{~mm}$, diameter of screw: $4.5 / 5.0 \mathrm{~mm}$ ).

the data of the two plates using the same specimen. The load-displacement data were analyzed for each condition to calculate stiffness, failure load, and displacement at failure. Stiffness was defined as the mean slope obtained from the linear part of the load-displacement curves of the three trials in the axial compression load test from $0 \mathrm{~N}$ to $550 \mathrm{~N}$. In the failure test, the mode of failure was recorded. Vertical displacement of the medial and lateral parts of the tibia at the osteotomy gap was recorded using a digital high-definition video camera in the failure test. The recorded images were transferred to a PC, and the medial and lateral vertical displacements at the osteotomy gap were measured using ImageJ software (National Institutes of Health, Bethesda, MD, USA) when total displacement of $2.5 \mathrm{~mm}$ was indicated.

\subsection{Statistics}

Statistical analysis was performed using paired and unpaired t-tests to analyze differences in stiffness, failure load, and vertical displacement between the Puddu and TomoFix plates or before and after HTO. Fisher's exact probability test was used for the analysis of the mode of failure in the failure test. The significance level was set at $\mathrm{P}<0.05$.

\section{Results}

No significant differences were observed in the measured mean values of stiffness for the intact bones between the Puddu and TomoFix groups (Figure 2). After HTO, the stiffness significantly $(\mathrm{P}<0.05)$ decreased to $30 \%$ of the intact bone in the Puddu group and $37 \%$ in the TomoFix group. No significant differences in stiffness after HTO were observed between the two groups.

The mean failure load was $1471.4 \mathrm{~N}$ and $1692.3 \mathrm{~N}$, and the total vertical displacement at failure was $3.1 \mathrm{~mm}$ and $2.9 \mathrm{~mm}$ in the Puddu and TomoFix groups, respectively. No significant differences were observed in the mean values of failure load and displacement at failure between the two groups. The failures resulted in bone fracture of the lateral spared cortex at the osteotomy level in all specimens, except for 1 specimen in the Puddu group. This specimen had lateral tibial plateau fracture at failure (Table 1).

Vertical displacement of the medial and lateral parts of the tibia at the osteotomy gap at $2.5 \mathrm{~mm}$ total displacement showed similar trend in the two groups (Figure 3). During axial compression loading, the displacements occurred mainly at the lateral osteotomy gap, while the medial gap was well preserved. No significant differences in displacement were observed between the two groups. 


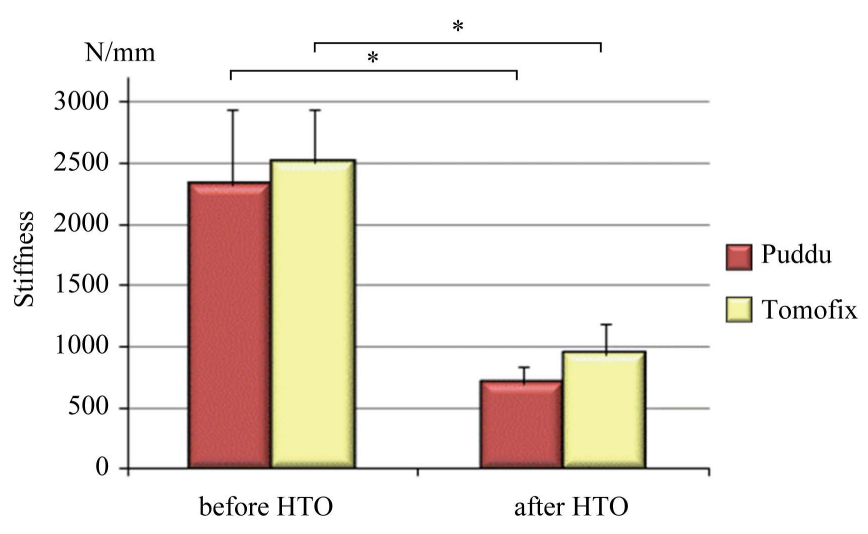

Figure 2. Mean stiffness of the specimens before and after openwedge HTO. Stiffness following HTO significantly decreased with both Puddu and TomoFix. No significant differences in stiffness were observed before and after HTO between the two groups. ${ }^{*} \mathrm{P}<0.05$.

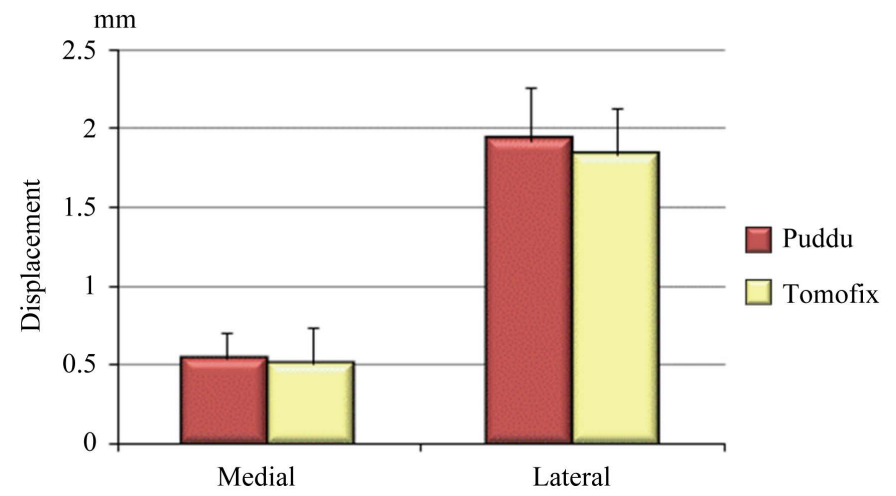

Figure 3. Vertical displacement of the medial and lateral parts of the tibia at the osteotomy gap at $2.5 \mathrm{~mm}$ total displacement. No significant differences in displacement were observed between Puddu and TomoFix.

Table 1. Results of the failure test. No significant differences were observed between Puddu and TomoFix plates.

\begin{tabular}{lccc}
\hline & Puddu & TomoFix & P value \\
\hline Failure load (N) (mean SD) & $1471.4(676.7)$ & $1692.3(577.8)$ & 0.82 \\
Displacement at failure (mm) (mean SD) & $3.1(1.0)$ & $2.9(0.8)$ & 0.18 \\
Mode of failure (number of specimen) & & 8 & 0.50 \\
Fracture of the lateral spared cortex at the osteotomy level & 7 & 0 & \\
Fracture of the lateral tibial plateau & 1 & & \\
\hline
\end{tabular}

\section{Discussion}

The open-wedge HTO procedure has several benefits, such as avoiding fibular osteotomy and potential complications from the dissection of the peroneal nerve or preserving the length of the lower extremity [3]. Stable fixation is required to avoid complications such as loss of correction or non-union, and implants specially designed for this procedure have been developed. The Puddu plate which is short with a block incorporated was one of these implants, and the previous version was a stainless short plate without a locking screw mechanism. Satisfactory clinical results were obtained [3]; however, complications, including implant failure and non-union, were also reported [4]. Recently, a new version of the titanium plate was developed with locking screws. TomoFix was also developed recently for open-wedge HTO. This plate is a longer and wider plate and an expected ri- 
gid fixation after osteotomy.

Several investigators have reported biomechanical studies of these locking plates. Agneskirchner et al. [5] compared the initial biomechanical stability of four different plates for open-wedge HTO. The tested plates were the Puddu plate, Puddu locking plate, long spacer locking plate, and TomoFix plate. Using each plate, axial compression loads were applied to Sawbones composite tibiae after HTO. Single load-to-failure tests as well as load-controlled cyclical failure tests were performed, and osteotomy gap motion was measured using linear displacement transducers. The results demonstrated that TomoFix showed higher primary stability compared with the shorter plates. Pape et al. [6] quantified the stabilizing effect of biplanar osteotomy versus uniplanar osteotomy using the Puddu locking plate and Tomofix plate in their cadaver study. Biplanar osteotomy was performed on each right tibia and uniplanar osteotomy was performed on each left tibia from the same specimen, with one tibia fixed with each locking plate. The results demonstrated that biplanar osteotomy increased the fixation stability significantly in anterioposterior and rotational planes in the axial compression test. The TomoFix plate showed higher primary stability compared with the Puddu locking plate, although the specimens utilized were "unpaired" in this comparison. Agneskirchner et al. [5] used composite tibial bone without the fibula. Pape et al. [6] used cadaver specimens and compared primary stability of bone to implant constructs between biplanar and uniplanar osteotomies using cadaveric tibia pairs. The specimens utilized were without the fibula, and the results of the stabilizing effect of the Puddu locking plate and TomoFix plate came from different specimens (nonpaired specimens).

We used paired specimens with the fibula to compare the stabilizing effect of the Puddu locking plate and TomoFix plate. One of the benefits of open-wedge HTO is avoidance of fibular osteotomy. Preserving the fibula in such a biomechanical study should be closer to clinical situation. After HTO, the mechanical axis and the loading point on the tibial surface of the knee joint shift laterally, and the role of the fibula to bear the body weight increases. No significant differences were observed between these two plates in terms of stiffness, failure load, and vertical displacement at the osteotomy gap in the axial compression test. Both the plates preserved the medial osteotomy gap during compression loading. The spacer inserted in the osteotomy gap was considered to increase stability, in addition to the longer and more rigid plate.

Spahn et al. [7] biomechanically investigated the stability of different internal fixations with or without a spacer in open-wedge HTO in a porcine bone model. The compared fixations were an angle stable and a nonangle stable plate with and without a spacer. They revealed that plates with a spacer had superior biomechanical properties, which supported our findings and considerations.

There were several limitations in this study. First, bone mineral density or biomechanical properties of the cadaveric specimens were not evaluated when comparing the biomechanical properties of two implants, although paired specimens were used in this study. Second, multiple loadings including rotational torque were not applied to the specimens. Third, the cadaveric specimens were elderly compared to the typical patients requiring HTO.

From the results of this study, we concluded that the Puddu and Tomofix plates had similar biomechanical properties in open-wedge HTO. The results of failure load and medial gap preservation indicated reliable stability after open-wedge HTO without fibular osteotomy. The result of the mode of failure test showed that a biomechanical weak point after this surgery might be the lateral spared cortex at the osteotomy level. Some modifications are applicable to this operation for better stability, such as stabilization of the lateral spared tibial cortex or gap filling with bone or materials.

\section{References}

[1] Floerkemeier, S., Staubli, A.E., Schroeter, S., Goldhahn, S. and Lobenhoffer, P. (2013) Outcome after High Tibial Open-Wedge Osteotomy: A Retrospective Evaluation of 533 Patients. Knee Surgery, Sports Traumatology, Arthroscopy, 21, 170-180. http://dx.doi.org/10.1007/s00167-012-2087-2

[2] Maffulli, N., Loppini, M., Longo, U.G., Denaro V. and Oliva, F. (2013) Bovine Xenograft Locking Puddu Plate versus Tricalcium Phosphate Spacer Non-Locking Puddu Plate in Opening-Wedge High Tibial Osteotomy: A Prospective Double-Cohort Study. International Orthopaedics, 37, 819-826. http://dx.doi.org/10.1007/s00264-013-1817-5

[3] Noyes, F.R., Mayfield, W., Barber-Westin, S.D., Albright, J.C. and Heckmann, T.P. (2006) Opening Wedge High Tibial Osteotomy. American Journal of Sports Medicine, 34, 1262-1273. http://dx.doi.org/10.1177/0363546505286144

[4] Nelissen, E.M., van Langelaan, E.J. and Nelissen, R.G. (2010) Stability of Medial Opening Wedge High Tibial Osteotomy: A Failure Analysis. International Orthopaedics, 34, 217-223. http://dx.doi.org/10.1007/s00264-013-1817-5 
[5] Agneskirchner, J.D., Freiling, D., Hurschler, C. and Lobenhoffer, P. (2006) Primary Stability of Four Different Implants for Opening Wedge High Tibial Osteotomy. Knee Surgery, Sports Traumatology, Arthroscopy, 14, 291-300. http://dx.doi.org/10.1007/s00167-005-0690-1

[6] Pape, D., Lorbach, O.L., Schmitz, C., Busch, L.C., van Giffen, N., Seil, R. and Kohn, D.M. (2010) Effect of a Biplanar Osteotomy on Primary Stability Following High Tibial Osteotomy: A Biomechanical Cadaver Study. Knee Surgery, Sports Traumatology, Arthroscopy, 18, 204-211. http://dx.doi.org/10.1007/s00167-009-0929-3

[7] Spahn, G., Mückley, T., Kahl, E. and Hofmann, G.O. (2006) Biomechanical Investigation of Different Internal Fixations in Medial Opening-Wedge High Tibial Osteotomy. Clinical Biomechanics, 21, 272-278.

http://dx.doi.org/10.1016/j.clinbiomech.2005.10.010 
Scientific Research Publishing (SCIRP) is one of the largest Open Access journal publishers. It is currently publishing more than 200 open access, online, peer-reviewed journals covering a wide range of academic disciplines. SCIRP serves the worldwide academic communities and contributes to the progress and application of science with its publication.

Other selected journals from SCIRP are listed as below. Submit your manuscript to us via either submit@scirp.org or Online Submission Portal.
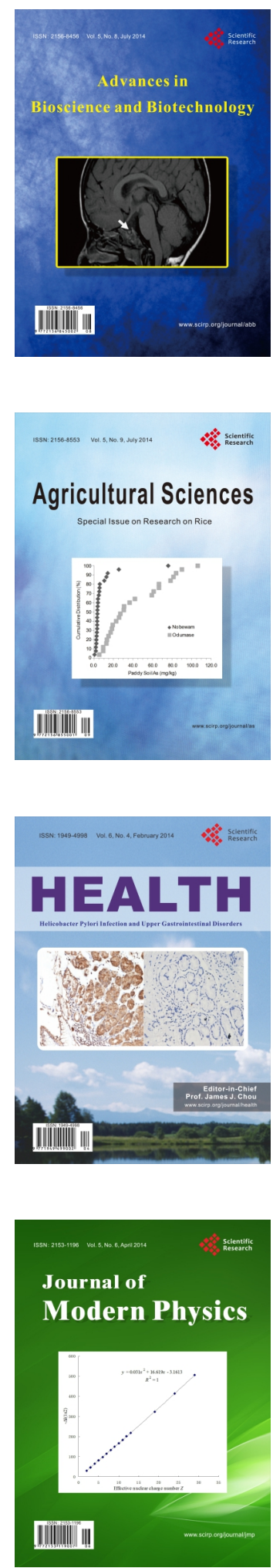
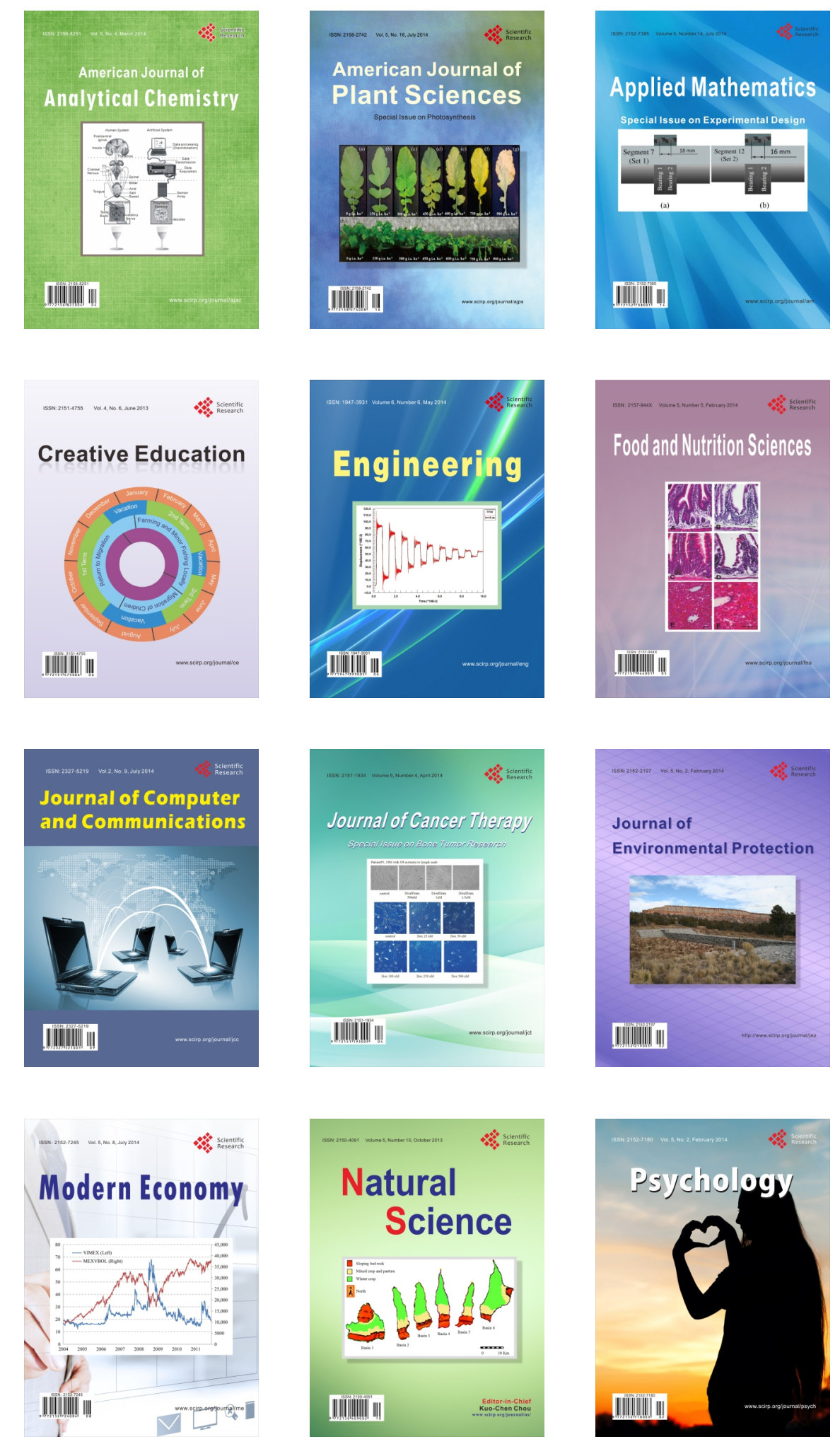